\section{THE DETERMINATION OF THE NEXT DOSE IN TUBERCULIN THERAPY*}

\section{MYER SOLIS-COHEN, A.B., M.D.} PHILADELPHIA

In the determination of the appropriate dose for the individual patient lie both the difficulty and the danger of tuberculin therapy. A dose in excess of the exact amount required frequently proves disastrous and has even hastened death. The possibilities of giving the wrong dose are increased by the very widle limits of tuberculin dosage, one patient requiring a dose one thousand or one million times that required by another patient, who is sometimes apparently of the same type.

There is no way of determining accurately the proper dose for a patient who has never taken tuberculin. The initial dose is always a matter of guess. Jixperience and judgment may indeed help one to guess more correctly, but they do not insure accuracy. White and Williams estimate the initial dose from the size of the areola produced by a von Pirquet test made with a definite amount of tuberculin; but they use this method only for incipient afebrile cases and the dosage they use I would be afraid to employ. My own rule with regard to the incipient dose is to give an amount that will probably do no harm. When beginning tuberculin treatment, therefore, I give, in incipient cases, one ten-millionth to one millionth of a milligram; in moderately advanced cases one billionth to one one-hundred-millionth of a milligram, and in far-advanced cases one trillionth to one billionth of a milligram. My reasons for giving this dosage I have explained at length in a previous communication. ${ }^{1}$

The dose for a patient who has already been given tuberculin, however, should not be determined by guess, but by careful estimation. It is here that judgment and experience count. From the effect produced by the last dose of tuberculin we can determine the amount to be given at the next dose. The dosage must be distinctly individual. It is impossible to generalize in tuberculin therapy or even to group our cases. There is no rule of thumb to guide us, nor any general plan of approximate dosage. We must study critically in each individual patient the phenomena that have followed the previous administrations of tuberculin, and from the data presented decide what the next dose in that case should be.

In order to pass an intelligent judgment it is important to have a clear idea as to the effect one desires from the use of tuberculin. There are really three methods of giving tuberculin. Sahli, Denys, and most of the German authorities, as well as Trudeau and his associates in this country, desire to accustom the patient gradually to large doses of tuberculin without causing any reaction in the process. Others, such as Koch, C. Spengler, Petruschky, Löwenstein, Möller and Pickert abroad, and White in this country, endeavor to give the dose that will just produce a slight reaction. The English school, including Latham and Inman, and Bullock and others in this country, aim to give the dose that has a favorable influence on the symptoms and to maintain this dose until it loses its effect. I am in thorough accord with this third group and partially agree and partially disagree with

* Read before the Philadelp iia Clinical Society, May 4, 1914.

1. Solis-Cohen, Myer: The Use of a Very Minute Initial Dose in Tuberculin Therapy, New York Med. Jour., 1913, xcviii, 268. the other two groups. This becomes possible if we distinguish between favorable and unfavorable reactions. I regard as signs of a favorable reaction: improvement in appetite, increase of strength, a feeling of well-being, lessening of the symptoms, flattening of the temperature curve, and all favorable phenomena occurring on the day tuberculin is given or on the day following. An tinfavorable reaction is shown by weakness, fatigue, drowsiness, headache, malaise, anorexia and increase of the various symptoms on the day of or on the day after tuberculin treatment. I try to give the dose that will produce a favorable reaction-which is really the object sought by Latham and his school. At the same time I half agree with those who desire to produce a reaction; only the reaction $I$ aim for is a favorable one. Inasmuch as I try to avoid producing an unfavorable reaction (unless very slight) I again half agree with those who would avoid reactions. This statement of the effects I seek to obtain and to avoid will explain the method I employ in determining the dosage.

The effect of tuberculin can usually be seen in the opsonic curve, the leukocytic count, the leukocytic differential picture, the Arneth count, the temperature curve, the subjective and the objective sumptoms and the physical signs. A study of all or of any one of these will give valuable information as to its action. Wright recommended that dosage be regulated by the effect on the opsonic index, but the difficulty and the errors in making the test and the fact that three or more determinations of the index each day are necessary for accuracy, render this method impracticable. Strickler and I, in a preliminary communication, ${ }^{2}$ suggested that tuberculin dosage be regulated by the effect on the Arneth and the lymphocytic curves, but we have not yet examined a sufficient number of cases to permit us to speak with any authority on this point. Dürel ${ }^{3}$ determines his dosage by its effect on the Arneth index, but as his results differ from those of Strickler and myself, all I can say is that in his hands it seems a safe and accurate guide. The time required for a differential count militates against its availability as a routine procedure, especially in the hands of the busy practitioner and among patients who cannot afford to pay for such laboratory tests. It is possible, on the other hand, by carefully studying the clinical symptoms and the temperature curve, to gain some knowledge as to the effect produced by each dose of tuberculin; and, indeed, Latham and Inman ${ }^{4}$ have shown that when a patient suffering from tuberculosis feels better and his symptoms show amelioration, his opsonic index is raised, and that a falling index is often associated with a peculiar frontal headache just above the eyebrows, with lassitude and a general feeling of malaise, with loss of appetite, a quickened pulse, increased expectoration, restlessness and inability to sleep. It has been my experience that slight clinical symptoms of an unfavorable reaction occur before there is any notable alteration in the temperature. These slight symptoms are of ten unnoted by the patient unless his attention is particularly

2. Solis-Cohen, Myer, and Strickler, Albert: The Effect of Tuberculin Treatment upon the Leukocytic Picture (a preliminary report), New York Med. Jour., 1912, xcv, 53.

3. Dürei, Wallace G.: The Polynuclear Neutrophil Leukocytic Count as a Guide for the Administration of Tuberculin. Tr. Nat. Ass'n for the Study and Prevention of Tuberculosis, 1912, p. 415.

4. Latham, Arthur, and Inman, A. C.: A Contribution to the Study of the Administration of Tuberculin in Pulmonary Tuberculosis, Lancet London, 1908, ii, 1280. 
called to them and sometimes can be elicited only by persistent questioning. In order not to miss this important evidence as to the effect of the doses already given, which must serve as a guide in the determination of the next dose, I always question the patient closely as to how he feels on the day of the tuberculin and on the day afterward, as compared with the days of the interval between doses. Of course one should avoid leading questions and should be careful not to suggest the answers. I ask, for instance, if the appetite on the day tuberculin is given is the same as, or better than, or worse than on the day preceding or on the day following the dose, or on the next day. Every new symptom and every change in an old symptom I put down as possible evidence of a reaction-favorable or unfavorable. To be on the safe side, in regulating the dosage I regard the occurrence of new unpleasant symptoms or the exacerbation of old symptoms on the day of or after the dose as probable evidence of an unfavorable reaction.

The next dose of tuberculin can be the same as or greater than or less than the last dose. In determining this dose I am guided by the following rules:

The next dose should be the same as the preceding close, when the latter was followed by a favorable reaction, such as a feeling of well-being, rise of spirits, increase of appetite, fall of an elevated temperature to normal, or reduction of the extent of the daily fluctuation; or when the preceding dose was followed by a very slight unfavorable reaction that lasted but a few hours, or at most a day, and was then followed by an improvement in the symptoms or general condition.

The next dose should be less than the preceding dose if the latter caused symptoms of an unfavorable reaction, such as rise of temperature, increase of symptoms, malaise, anorexia, pains, loss of weight, etc. (with the one exception just mentioned), or if definite and prolonged painfulness and inflammation occurred at the site of injection-the so-called skin reaction. If the rise of temperature and the other symptoms lasted but a few hours, the dose should be reduced slightly-a half, for instance. If the rise and the symptoms lasted all day, the next dose should be one-tenth, or less, of the preceding dose. When the reaction has been marked or has lasted several days in spite of rest, the next dose should be one hundredth to one thousandth of the last dose.

When the last dose has had no effect of any kind, it should be increased at the next administration. liven in febrile cases in which no change one way or the other is noted in temperature or symptoms, the dose should be increased until signs of a favorable or an unfavorable reaction appear. When the effect of a hitherto appropriate dose is passing off, as shown by the last close being followed by a rise to a higher level of a temperature which has been kept down, or the reappearance of more marked daily fluctuations and an increase of symptoms which had been ameliorated, the next dose should be increased.

The rate of increase in ordinary cases should be about 50 per cent. of the preceding dose. The course of tuberculin so commonly given in series of nine doses beginning with 100 per cent. increase and ending with an 11 per cent. increase, followed again by 100 per cent. increase in the next series, I consider dangerous. The scheme is represented thus: $1,2,3$, $4,5,6,7,8,9,10,20,30$, etc. If a scheme is desircd the following is much safer: $1,1.5,2,3,5,7,10,15$, 20 , 30 , etc. Occasionally, when a very minute initial dose is given without the slightest effect, a more rapid rate of increase-not exceeding 100 per cent.may be justified, provided the patient is closely watched and quizzed for the first appearance of a slight reaction. In patients who have had to have their dose reduced on account of a reaction the subsequent increase or dose should be made very gradually and cautiously, from 10 to 25 per cent. of the preceding dose being sufficient increase.

The length of the interval between doses requires consideration. Ordinarily, in patients who have shown no effect of any kind from tuberculin and in those who are doing well on it, an interval of seven, five, or even three days is usually satisfactory. It is best to begin with a seven days' interval. In advanced cases and in susceptible and nervous individuals, and with the larger doses, ten days would often be better. After an unfavorable reaction one must wait until all the reactive symptoms have disappeared. If the reaction has been severe it is best to postpone the next dose until a week or more after the disappearance of all symptoms. When the depression of the temperature curve lasts but three days and then rises until the next dose is given, or when the symptoms that have been ameliorated return after three days, the next dose should be given at the end of three days, just before the effect of the preceding dose has passed away. One can often tell from the reappearance of fever and other symptoms about the length of time one dose of tuberculin exerts its influence and can then give the next dose as the effect of the last is wearing off.

The dosage and the interval are of the utmost importance in the use of this dangerous remedy. $\Lambda$ physician who does not wish to give the time and attention their determination demands, should never ariminister tuberculin. Otherwise he cannot obey IIippocrates' injunction to do good or do no harm.

\section{COLLARGOL IN PYELOGRAPHY}

WITH REPORT OF AN INTERESTING CASE

ANDREW J. CROWELL, M.D.

CHARLOTTE, N. C.

In looking up the literature on the subject, I find but few mortalities reported following the use of collargol, but a number of cases in which great damage has been done the kidney as shown by subsequent operation. The pathologic findings in these cases have been similar but the theories as to the cause of the destructive action of the remedy differ with the various observers.

There is also great divergence of opinion as to the toxicity of this drug; some claiming that it has an irritating and even cauterizing effect, while others assert that it has no injurious effect whatever on the pelvic mucosa, the tubular epithelium or other tissues of the kidney. There are still others who seem to be of the opinion that the kidney cells have the power of ingesting and distributing the drug throughout the entire kidney, in other words, the theories are that the drug may be distributed by absorption or by active diffusion under pressure.

From my own observations, I am of the opinion that the injurious results following the use of collargol in pyelography are from a diffusive process under pres- 